\title{
Revision of conjectures about the opponent's utilities in signaling games
}

Citation for published version (APA):

Schulteis, T. J. W., Perea ý Monsuwé, A., Peters, H. J. M., \& Vermeulen, A. J. (2004). Revision of conjectures about the opponent's utilities in signaling games. METEOR, Maastricht University School of Business and Economics. METEOR Research Memorandum No. 008 https://doi.org/10.26481/umamet.2004008

Document status and date:

Published: 01/01/2004

DOI:

10.26481/umamet.2004008

Document Version:

Publisher's PDF, also known as Version of record

\section{Please check the document version of this publication:}

- A submitted manuscript is the version of the article upon submission and before peer-review. There can be important differences between the submitted version and the official published version of record.

People interested in the research are advised to contact the author for the final version of the publication, or visit the DOI to the publisher's website.

- The final author version and the galley proof are versions of the publication after peer review.

- The final published version features the final layout of the paper including the volume, issue and page numbers.

Link to publication

\footnotetext{
General rights rights.

- You may freely distribute the URL identifying the publication in the public portal. please follow below link for the End User Agreement:

www.umlib.nl/taverne-license

Take down policy

If you believe that this document breaches copyright please contact us at:

repository@maastrichtuniversity.nl

providing details and we will investigate your claim.
}

Copyright and moral rights for the publications made accessible in the public portal are retained by the authors and/or other copyright owners and it is a condition of accessing publications that users recognise and abide by the legal requirements associated with these

- Users may download and print one copy of any publication from the public portal for the purpose of private study or research.

- You may not further distribute the material or use it for any profit-making activity or commercial gain

If the publication is distributed under the terms of Article $25 \mathrm{fa}$ of the Dutch Copyright Act, indicated by the "Taverne" license above, 


\title{
Revision of conjectures about the opponent's utilities in signaling games
}

\author{
Tim Schulteis* Andres Perea* Hans Peters* \\ Dries Vermeulen*
}

March 2004 (this version)

\begin{abstract}
In this paper we apply the concept of preference conjecture equilibrium introduced in Perea (2003) to signaling games and show its relation to sequential equilibrium. Furthermore, we introduce the concept of minimum revision equilibrium and show how this can be interpreted as a refinement of sequential equilibrium. We also present a method to compute preference conjecture equilibria.
\end{abstract}

\section{Introduction}

In this paper we deal with the question what player 2 in a signaling game should believe if he observes a message which he did not expect. Usually, the following assumptions are made:

(i) each player has a utility function that is known to all players throughout the game;

(ii) all players are rational utility maximizers.

In the concept of Nash equilibrium (see Nash, 1950) it is required that each player plays optimally given the strategy of his opponent. In the concept of sequential equilibrium (Kreps and Wilson, 1982) it is additionally required that player 2 has beliefs on information sets that are not reached in equilibrium and decides optimally given these beliefs. However, in signaling games sequential equilibrium does not put any further restrictions on these

* Department of Quantitative Economics, University of Maastricht, P.O. Box 616, 6200 MD Maastricht, The Netherlands. Telephone: +31-43-3883835. Telefax: +31-43-3884874. Email addresses: T.Schulteis@ke.unimaas.nl, A.Perea@ke.unimaas.nl, H.Peters@ke.unimaas.nl, D.Vermeulen@ke.unimaas.nl. 
beliefs. In order to make the concept more powerful, several refinements were introduced in literature, such as perfect sequential equilibrium (Grossman and Perry, 1986), the intuitive criterion (Cho and Kreps, 1987) and divine equilibrium (Banks and Sobel, 1987). These refinements put restrictions on player 2's beliefs on information sets that are not reached in equilibrium. In all these refinements, the idea is that player 2, upon observing an unexpected message, makes a distinction between "less plausible" and "more plausible" types, and attaches positive probability only to the more plausible types. Throughout this reasoning process the utility functions are assumed to be fixed, which implies that player 2, upon observing an unexpected message, cannot revise his beliefs about player 1's utility function.

We will follow an alternative path, namely to insist on a player's belief of having a rational opponent and to assume that player 2 has a conjecture about his opponent's utility function, which he may revise after observing player 1's message. This revision should be such that the given message becomes optimal for player 1 . This leads to the concept of preference conjecture equilibrium, first formalized in Perea (2003).

It is important at this stage to compare preference conjecture equilibrium to rationality concepts that have been defined for situations where beliefs about the opponent's utility functions cannot be revised. As a benchmark for the latter, consider the concept of sequential equilibrium. In the concept of sequential equilibrium applied to signaling games, player 2 has beliefs about player 1's types. In contrast, in the concept of preference conjecture equilibrium player 2 holds, at the beginning of the game and after observing player 1's message, a belief about his opponent's utility function as well as a belief about the opponent's strategy choice. Furthermore, we demand that player 1's expectation about player 2's strategy choice coincides with player 2's strategy choice. When player 2 observes a message sent by his opponent that would have been suboptimal for player 1 given player 2's expectations at the beginning of the game, player 2 revises his belief about player 1's utility function. These changes are inevitable once we insist on the assumption that the players are rational and expect their opponent to be rational too. The profile of beliefs that emerges from this procedure, for every player on every information set, is called a conjecture profile.

We show that for signaling games the concepts of preference conjecture equilibrium and sequential equilibrium are in some sense equivalent. As an illustration, consider the game in Figure 1. This is the well known beer and quiche game introduced by Cho and Kreps (1987). At the beginning of the game nature decides between weak $(w)$ and strong $(s)$ as types for player 1 with probabilities 0.1 and 0.9 respectively. Then player 1 chooses between 


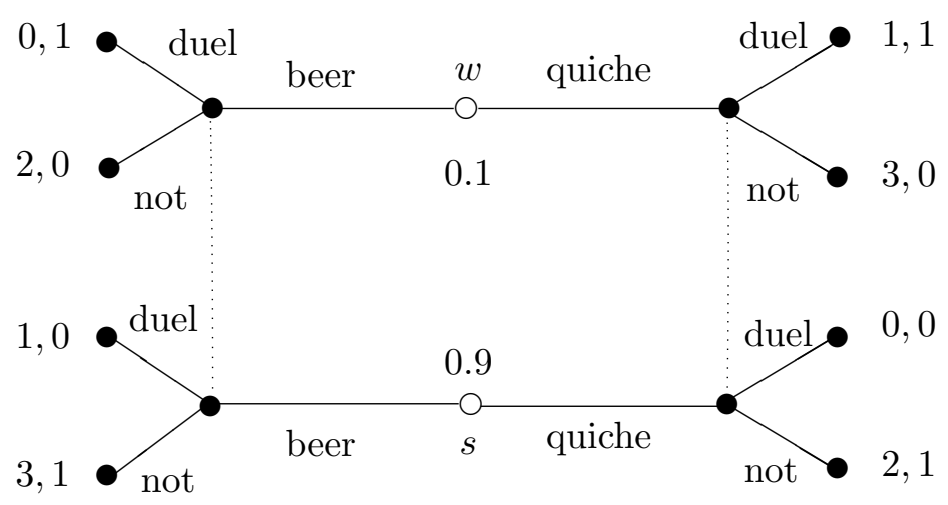

Figure 1: The beer and quiche game

the messages beer and quiche. Player 2 observes this chosen message but not player 1's type. Based on his observation he subsequently decides to either challenge player 1 for a duel or not. This game possesses two kinds of sequential equilibria, namely one in which every type of player 1 chooses beer and one in which player 1 always chooses quiche. The intuitive criterion (Cho and Kreps, 1987) excludes equilibria of the second kind by the following argument. If player 2 expects both types to choose quiche, then he chooses not to duel after quiche. But what if player 2 observes beer? He concludes that it is the strong type that deviated, since the weak type can get at most 2 by deviating, which is less than 3 , which he gets by choosing quiche; and the strong type could get 3 by deviating, which is more than the 2 he gets by choosing quiche. Hence, player 2 will choose to avoid the duel after observing beer. Then however, the strong type will deviate from the equilibrium by choosing beer. Hence, the intuitive criterion excludes the second type of equilibrium, because it is supported by the belief that player 1 is weak if he drinks beer, in spite of the fact that the weak type always obtains less than its equilibrium payoff 3 by deviating. To illustrate the concept of preference conjecture equilibrium, we now consider the depicted utilities as the players' conjectures about their opponent's utility function at the beginning of the game. Furthermore we let the conjectures about the opponent's strategy choice be such that player 2 initially believes that both types of player 1 will choose quiche and player 1 expects that player 2 will duel after observing beer and not duel after observing quiche. So the question is: what should player 2 decide if he observes beer? By deviating from player 2's expectation, the weak type now has a utility of 0 , compared to 3 if he would have chosen quiche. In order to rationalize this, we might 
assume that player 2 thinks the weak type's payoff will be 4 after deviating. Thus player 2 will think the weak type's preference relation is such that the weak type prefers beer over quiche, in contrast to what he believed at the beginning of the game. Of course, player 2 may also believe that only the strong type has chosen beer after observing the message beer and may thus choose not to duel. But in that case the strong type would improve his utility by deviating from the initial conjecture and we will show that these conjectures cannot be extended to a preference conjecture equilibrium. To illustrate the relationship with sequential equilibrium, we see that both types taking quiche can only lead to an equilibrium if player 2, after observing beer, believes it was the weak type that deviated with a probability of at least 0.5. Thus player 2 might also believe that both types had some incentive to deviate, since he also underestimated the strong type's payoff after duelling and getting his favorite breakfast. Because of the same argument, he will have to choose to duel, so his conjecture about player 1's utility function after beer and duel might be 4 for the weak type and 5 for the strong type. It is, however, very intuitive to impose the condition that the revisions should be as limited as possible, which means that the revised conjectures should be as close as possible to the initial conjecture. The question arises how to measure the revisions and what the requirement of minimality of these revisions implies.

In this paper we introduce the concept of minimum revision equilibrium, a refinement of preference conjecture equilibrium that imposes further restrictions on the belief revisions based on the revision index. Consider again the game in Figure 1. In games with only two messages we consider the number of types for which player 2 changes his belief about their preference relation over messages. In the preference conjecture equilibrium where after observing beer, player 2 believes that only the weak type has chosen beer, player 2 has to change his belief about only the weak type's preference relation over beer and quiche. In the second preference conjecture equilibrium, where we assumed that after observing beer, player 2 believes that both types have chosen beer, player 2 has to change his belief over both types' preference relations over beer and quiche. We thus say that the revision indices are 1 and 2 respectively. In order to determine the minimum revision equilibrium, we compute this index for all preference conjecture equilibria and choose a preference conjecture equilibrium with the minimal index. Since preference conjecture equilibrium will be shown to coincide with sequential equilibrium for signaling games, this implies that minimum revision equilibrium can be interpreted as a refinement of sequential equilibrium. We find in the example above that there are two minimum revision 
equilibria, both with a revision index of one. The first one corresponds to the sequential equilibrium in which both types choose quiche and player 2, when observing beer, attaches probability one to the fact that the weak type deviated. The second minimum revision equilibrium corresponds to the sequential equilibrium in which both types choose beer and, after observing quiche, player 2 attaches probability one to the fact that the weak type deviated. We see that one of these minimum revision equilibria coincides with the equilibrium that is selected by the intuitive criterion, but the other one does not.

The paper is organized as follows. In Section 3 we develop the concept of preference conjecture equilibrium for signaling games. In Section 4 we investigate the relationship with sequential equilibrium, established by Theorems 4.1 and 4.3. We show that every preference conjecture equilibrium induces a sequential equilibrium and that vice versa every sequential equilibrium corresponds to a preference conjecture equilibrium. In Section 5 we introduce the minimum revision equilibrium and elaborate on its application as a refinement of sequential equilibrium in signaling games. In Section 6 we provide some examples and compare the concept of minimum revision equilibrium to the intuitive criterion. Section 7 elaborates on the structure of the set of sequential equilibria in signaling games, written as a union of sets, on which the revision index is constant. These sets can be computed by solving systems of linear inequalities, which facilitates the computation of minimum revision equilibria.

\section{Preliminaries}

In this section we define signaling games, and Nash and sequential equilibrium in signaling games. We also introduce an illustrative example that is used throughout the paper.

Notation. For a finite set $Q, \Delta(Q)$ denotes the set of all probability distributions over $Q$, and $\Delta^{0}(Q)$ denotes the set of probability distributions over $Q$ which assign positive probability to every $q \in Q$.

Definition 2.1 A signaling game is a 6-tuple $\mathcal{S}=\left(T, M, A, p, u_{1}, u_{2}\right)$ where $T, M$ and $A$ are finite sets; $p \in \Delta^{0}(T)$; and $u_{1}: T \times M \times A \rightarrow \mathbb{R}$ and $u_{2}: T \times M \times A \rightarrow \mathbb{R}$ are functions.

A signaling game is played as follows: 


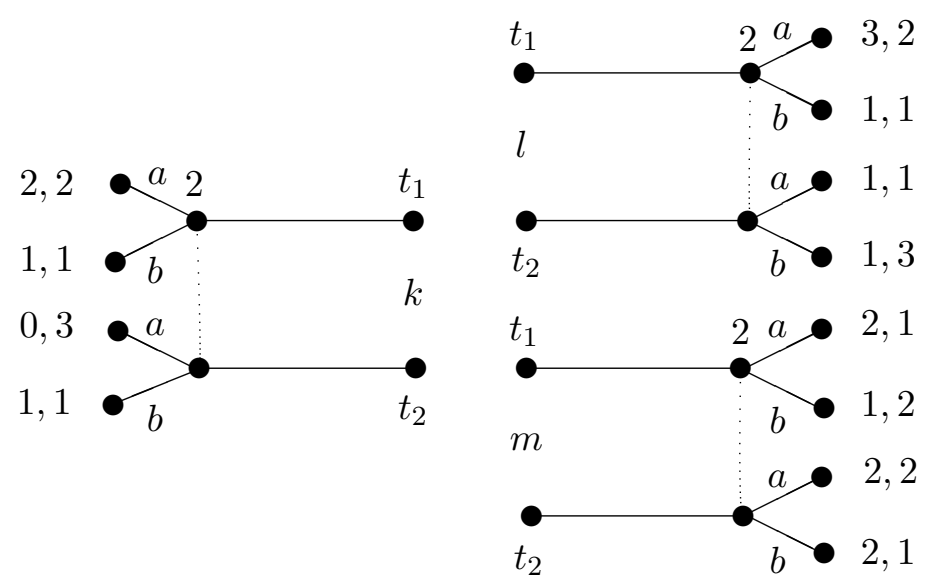

Figure 2: Example 2.2

- Nature selects a type $t$ of player 1 from the set $T$ of types. Type $t$ is selected with probability $p(t)>0$.

- Player 1 , knowing his type, chooses a message $m$ from the set of messages $M$.

- Player 2, not knowing the type of player 1, but knowing the probability distribution $p$, observes the message $m$ and subsequently chooses an action a from the set $A$.

- The utilities are $u_{1}(t, m, a)$ for player 1 and $u_{2}(t, m, a)$ for player 2 .

In the usual extensive form representation of a signaling game, each decision node of player 1 corresponds to a type $t$. Each decision node of player 2 corresponds to a pair $(t, m)$. A terminal node corresponds to a triple $(t, m, a)$. An information set of player 2 corresponds to a message $m$. Henceforth, we use the notation fixed in Definition 2.1 for a signalling game, unless stated otherwise.

The following example is used for illustrative purposes throughout the paper.

Example 2.2 In this game (see Figure 2) player 1 can be of two types $t_{1}$ and $t_{2}$, each with probability $\frac{1}{2}$. Player 1 chooses between three messages $k$, $l$, and $m$, and player 2 chooses between the actions $a$ and $b$. The numbers at the terminal nodes are the utilities to players 1 and 2 , respectively. 
A pure strategy for player 1 in a signaling game is a map $s_{1}: T \rightarrow M$, defining a message $m$ for every type $t$. The set of pure strategies for player 1 is denoted by $S_{1}$. An element $\sigma_{1} \in \Delta\left(S_{1}\right)$ is called a mixed strategy for player 1 . So, $\sigma_{1}\left(s_{1}\right)$ is the probability of the pure strategy $s_{1}$. Similarly, a pure strategy for player 2 is a map $s_{2}: M \rightarrow A$, defining an action $a$ for every message $m$. The set of pure strategies for player 2 is denoted by $S_{2}$. An element $\sigma_{2} \in \Delta\left(S_{2}\right)$ is called a mixed strategy for player 2. So, $\sigma_{2}\left(s_{2}\right)$ is the probability of the pure strategy $s_{2} \cdot{ }^{1}$

A system of beliefs (for player 2) is a collection $\beta=(\beta(m))_{m \in M}$ where $\beta(m)=(\beta(t \mid m))_{t \in T} \in \Delta(T)$ for each $m \in M$. As usual, $\beta(t \mid m)$ is the probability that player 2 attaches to player 1 being of type $t$ if message $m$ is played. For a mixed strategy $\sigma_{1} \in \Delta\left(S_{1}\right)$ let

$$
\sigma_{1}(m \mid t):=\sum_{s_{1} \in S_{1}: s_{1}(t)=m} \sigma_{1}\left(s_{1}\right)
$$

be the probability that player 1 plays $m$ if he is of type $t$. The pair $\left(\sigma_{1}, \beta\right)$ is called Bayesian consistent ${ }^{2}$ if for all types $t$

$$
\beta(t \mid m)=\frac{p(t) \cdot \sigma_{1}(m \mid t)}{\sum_{t^{\prime} \in T} p\left(t^{\prime}\right) \cdot \sigma_{1}\left(m \mid t^{\prime}\right)}
$$

for all messages $m$ such that $\sum_{t^{\prime} \in T} p\left(t^{\prime}\right) \cdot \sigma_{1}\left(m \mid t^{\prime}\right)$ is positive. So Bayesian consistency puts no restrictions on beliefs on information sets that are reached with probability zero if $\sigma_{1}$ is played.

A triple $\left(\sigma_{1}, \sigma_{2}, \beta\right) \in \Delta\left(S_{1}\right) \times \Delta\left(S_{2}\right) \times \Delta(T)^{M}$ is called an assessment. For such an assessment

$$
U_{1}\left(t, s_{1}, \sigma_{2}\right):=\sum_{s_{2} \in S_{2}} \sigma_{2}\left(s_{2}\right) \cdot u_{1}\left(t, s_{1}(t), s_{2}\left(s_{1}(t)\right)\right)
$$

is the expected utility for player 1 if his type is $t$, he plays $s_{1}$ and player 2 plays $\sigma_{2}$, and

$$
U_{2}\left(m, s_{2}, \beta\right):=\sum_{t \in T} \beta(t \mid m) \cdot u_{2}\left(t, m, s_{2}(m)\right)
$$

is the expected utility for player 2 if he observes message $m$, has belief $\beta$ and plays $s_{2}$. We call $s_{1} \in S_{1}$ optimal with respect to $\sigma_{2}$ if

$$
U_{1}\left(t, s_{1}, \sigma_{2}\right) \geq U_{1}\left(t, s_{1}^{\prime}, \sigma_{2}\right)
$$

\footnotetext{
${ }^{1}$ For our purposes, it is convenient to work with mixed strategies instead of behavioral strategies. In Section 6 we also use behavioral strategies.

${ }^{2}$ In signaling games, Bayesian consistency is equivalent to consistency as defined in Kreps and Wilson (1982).
} 
for all $s_{1}^{\prime} \in S_{1}$ and all types $t$. For $m \in M$, we call $s_{2} \in S_{2}$ optimal with respect to $\beta$ on $m$ if

$$
U_{2}\left(m, s_{2}, \beta\right) \geq U_{2}\left(m, s_{2}^{\prime}, \beta\right)
$$

for all $s_{2}^{\prime} \in S_{2}$. We call $s_{2} \in S_{2}$ optimal with respect to $\beta$ if $s_{2}$ is optimal with respect to $\beta$ on every $m \in M$.

We say that $\sigma_{1}$ is optimal with respect to $\sigma_{2}$ if, for all $s_{1} \in S_{1}, \sigma_{1}\left(s_{1}\right)>0$ implies that $s_{1}$ is optimal with respect to $\sigma_{2}$. We call $\sigma_{2}$ optimal with respect to $\sigma_{1}$ if, for all $s_{2} \in S_{2}$ and all $m \in M$ with $\sum_{t^{\prime} \in T} p\left(t^{\prime}\right) \sigma_{1}\left(m \mid t^{\prime}\right)>0$, $\sigma_{2}\left(s_{2}\right)>0$ implies that $s_{2}$ is optimal with respect to $\beta$ on $m$, where $\beta$ satisfies (1). We call $\sigma_{2}$ optimal with respect to $\beta$ if, for all $s_{2} \in S_{2}, \sigma_{2}\left(s_{2}\right)>0$ implies that $s_{2}$ is optimal with respect to $\beta$.

Definition 2.3 The assessment $\left(\sigma_{1}, \sigma_{2}, \beta\right)$ is a (Bayesian) Nash equilibrium if

(i) $\left(\sigma_{1}, \beta\right)$ is Bayesian consistent;

(ii) $\sigma_{1}$ is optimal with respect to $\sigma_{2}$;

(iii) $\sigma_{2}$ is optimal with respect to $\sigma_{1}$.

If, moreover, $\sigma_{2}$ is optimal to $\beta$, then $\left(\sigma_{1}, \sigma_{2}, \beta\right)$ is a sequential equilibrium. ${ }^{3}$

The difference between (Bayesian) Nash (cf. Nash, 1950, 1951; Harsanyi, 1967, 1968) and sequential (cf. Kreps and Wilson, 1982) equilibrium is that in the latter player 2 also plays optimally on information sets reached with zero probability.

\section{Preference conjecture equilibrium}

The concept of preference conjecture equilibrium was first introduced in Perea (2003) for general games in extensive form. In the present paper we study this concept for signaling games. ${ }^{4}$

In a conjecture profile, each player has a conjecture about the opponent's strategy choice and a conjecture about the opponent's utility function. For

\footnotetext{
${ }^{3}$ In the literature this form of optimality is also called sequential rationality, see Kreps and Wilson (1982).

${ }^{4}$ In Perea (2003) the variant of preference conjecture equilibrium that we discuss here is called weak 'preference conjecture equilibrium'.
} 
player 1 , these conjectures are made at the beginning of the game: since player 1 moves first and only once, it makes no sense to consider revisions of player 1's conjectures. For player 2 the situation is different. Player 2 holds conjectures at the beginning of the game but may revise these after observing player 1's move. A conjecture profile entails induced conjectured preferences of the players. In a preference conjecture equilibrium the players believe each other to play rationally - that is, to maximize their conjectured preferences -implying that player 2 may have to revise his conjectures about player 1. Furthermore, revisions of conjectures about player 1's strategies will be restricted by imposing Bayesian updating.

Formally, starting with player 1 , let $\mu_{12} \in \Delta\left(S_{2}\right)$ be his conjecture (at the beginning of the game) about player 2's strategy choice. Thus, $\mu_{12}\left(s_{2}\right)$ is the probability player 1 assigns to player 2 playing strategy $s_{2}$. Similarly, let $u_{12}: T \times M \times A \rightarrow \mathbb{R}$ be player 1's conjecture about player 2's utility function. Denote $c_{12}=\left(\mu_{12}, u_{12}\right)$.

As explained above, for player 2 the situation is different. He holds conjectures both at the beginning of the game and at every information set $m \in M$ : depending on the observed message, player 2 may revise the conjecture held at the beginning. To denote conjectures of player 2 at the beginning of the game it is convenient to introduce the symbol $h_{0}$. Thus, for the beginning of the game we denote $S_{1}\left(h_{0}\right):=S_{1}$ and for $m \in M$ we denote $S_{1}(m):=\left\{s_{1} \in S_{1} \mid\right.$ there exists $t \in T$ with $\left.s_{1}(t)=m\right\}$. Let $M^{*}:=$ $M \cup\left\{h_{0}\right\}$. For all $m \in M^{*}$, let $\mu_{21}(m) \in \Delta\left(S_{1}(m)\right)$ be player 2's conjecture at $m$ about player 1's strategy choice. For $s_{1} \in S_{1}(m), \mu_{21}(m)\left(s_{1}\right)$ is the probability player 2 assigns at $m$ to player 1 playing $s_{1}$. Similarly, for all $m \in$ $M^{*}$ let $u_{21}(m): T \times M \times A \rightarrow \mathbb{R}$ be player 2's conjecture at $m$ about player 1 's utility function. For all $m \in M^{*}$ denote $c_{21}(m)=\left(\mu_{21}(m), u_{21}(m)\right)$.

Definition 3.1 A combination $c:=\left(c_{12},\left(c_{21}(m)\right)_{m \in M^{*}}\right)$ is called a conjecture profile.

In a preference conjecture equilibrium a conjecture profile plays a role similar to that of an assessment in a sequential equilibrium. Also for a conjecture profile we introduce optimality conditions. We start, however, with a condition implying that player 2 revises his conjecture about player 1 's strategy according to Bayes' rule, whenever possible.

Definition 3.2 A conjecture profile satisfies Bayesian updating if

$$
\mu_{21}(m)\left(s_{1}\right)=\frac{\mu_{21}\left(h_{0}\right)\left(s_{1}\right)}{\sum_{s_{1}^{\prime} \in S_{1}(m)} \mu_{21}\left(h_{0}\right)\left(s_{1}^{\prime}\right)}
$$


for all $s_{1} \in S_{1}(m)$ and all $m \in M$ such that $\sum_{s_{1}^{\prime} \in S_{1}(m)} \mu_{21}\left(h_{0}\right)\left(s_{1}^{\prime}\right)>0$.

The next task is to define optimality on the parts of both players in a conjecture profile. For this we use that, given their conjectures, players can compute their opponent's expected utility.

Let $m \in M^{*}$. Player 2 can compute his conjecture at $m$ about player 1's expected utility if player 1 has type $t$, conjecture $\mu \in \Delta\left(S_{2}\right)$ about player 2 's strategy choice, utility function $u_{21}(m)$, and plays $s_{1}$, as

$$
U_{21}\left(t, s_{1}, u_{21}(m), \mu\right):=\sum_{s_{2} \in S_{2}} \mu\left(s_{2}\right) \cdot u_{21}(m)\left(t, s_{1}(t), s_{2}\left(s_{1}(t)\right)\right) .
$$

In order to justify the next step in our argument we make the assumption that conjectures are common belief among the players. This means that a player's (informal) conjecture about an opponent's conjecture coincides with this opponent's conjecture. In particular, this implies that player 2's conjecture about player 1's conjecture about player 2's strategy choice coincides with player 1's conjecture about player 2's strategy choice. Therefore it makes sense to evaluate the above conjecture of player 2 at $m$ about player 1 's expected utility at $\mu=\mu_{12}$. Thus we write

$$
U_{21}\left(t, s_{1}, u_{21}(m), \mu_{12}\right)=\sum_{s_{2} \in S_{2}} \mu_{12}\left(s_{2}\right) \cdot u_{21}(m)\left(t, s_{1}(t), s_{2}\left(s_{1}(t)\right)\right) .
$$

A strategy $s_{1}$ is optimal for player 1 with respect to $\mu_{12}$ and $u_{21}(m)$ if

$$
U_{21}\left(t, s_{1}, u_{21}(m), \mu_{12}\right) \geq U_{21}\left(t, s_{1}^{\prime}, u_{21}(m), \mu_{12}\right)
$$

for all $s_{1}^{\prime} \in S_{1}$ and all $t \in T$.

Similarly, consider a pure strategy $s_{2}$ of player 2 , a utility function $u_{12}$ as well as a probability distribution $\mu^{\prime} \in \Delta\left(S_{1}(m)\right.$ ), where now $m \in M$ (so $m \neq h_{0}$ : see Remark 3.4 below). Then player 1 holds a conjecture about player 2's expected utility at $m$ given by

$$
U_{12}\left(m, s_{2}, u_{12}, \mu^{\prime}\right):=\sum_{t \in T} p(t) \sum_{s_{1}: s_{1}(t)=m} \mu^{\prime}\left(s_{1}\right) \cdot u_{12}\left(t, m, s_{2}(m)\right) .
$$

Again because of the assumption of common belief of conjectures it makes sense to evaluate this at $\mu^{\prime}=\mu_{21}(m)$ :

$$
U_{12}\left(m, s_{2}, u_{12}, \mu_{21}(m)\right)=\sum_{t \in T} p(t) \sum_{s_{1}: s_{1}(t)=m} \mu_{21}(m)\left(s_{1}\right) \cdot u_{12}\left(t, m, s_{2}(m)\right) .
$$


The strategy $s_{2}$ is optimal for player 2 with respect to $\mu_{21}(m)$ and $u_{12}$ if

$$
U_{12}\left(m, s_{2}, u_{12}, \mu_{21}(m)\right) \geq U_{12}\left(m, s_{2}^{\prime}, u_{12}, \mu_{21}(m)\right)
$$

for all $s_{2}^{\prime} \in S_{2}$.

Definition 3.3 A conjecture profile $c$ satisfies optimality if:

1. for all $m \in M^{*}$ and for all $s_{1} \in S_{1}$, if $\mu_{21}(m)\left(s_{1}\right)>0$, then $s_{1}$ is optimal for player 1 with respect to $\mu_{12}$ and $u_{21}(m)$;

2. for all $m \in M$ and for all $s_{2} \in S_{2}$, if $\mu_{12}\left(s_{2}\right)>0$, then $s_{2}$ is optimal for player 2 with respect to $\mu_{21}(m)$ and $u_{12}$.

Remark 3.4 Observe that, in this definition, optimality on the part of player 2 is not required on $h_{0}$, that is, at the beginning of the game: it is not hard to verify that this is taken care of by the second part of the definition combined with Bayesian updating.

Now, we are sufficiently equipped to define the main concept of this paper.

Definition 3.5 A conjecture profile $c$ is a preference conjecture equilibrium if it satisfies optimality and Bayesian updating.

Example 3.6 As an illustration, consider Example 2.2. Consider the conjecture profile $c$, where $\mu_{12}$ attaches probability 1 to strategy $(a, a, a)$ and $u_{12}$ coincides with player 2's utilities in Figure 2. Let $\mu_{21}\left(h_{0}\right), \mu_{21}(l)$ and $\mu_{21}(m)$ attach probability 1 to strategy $(l, m)$, and let $\mu_{21}(k)$ attach probability 1 to strategy $(k, m)$. Let $u_{21}\left(h_{0}\right), u_{21}(l)$ and $u_{21}(m)$ coincide with player 1's utilities in Figure 2, and let $u_{21}(k)\left(t_{1}, k, a\right)=5$ and $u_{21}(k)\left(t^{\prime}, m^{\prime}, a^{\prime}\right)=$ $u_{21}\left(h_{0}\right)\left(t^{\prime}, m^{\prime}, a^{\prime}\right)$ for all $\left(t^{\prime}, m^{\prime}, a^{\prime}\right) \neq\left(t_{1}, m, a\right)$. This conjecture profile is actually a preference conjecture equilibrium and can be interpreted as follows. Given his conjecture $\mu_{21}\left(h_{0}\right)$, player 2 initially expects message $k$ not to be chosen. However, if he observes message $k$, he revises his conjecture about player 1's strategy choice and utility function in such a way that the choice $k$ becomes optimal for at least one of the types of player 1. In this conjecture profile $c$, player 2 does so by raising the utility for type $t_{1}$ after message $k$ and action $a$, while keeping all other utilities unchanged. Thus player 2, after observing message $k$, believes that it was type $t_{1}$ and not $t_{2}$ who sent this message. Hence player 2, after observing $k$, assigns probability 1 to the strategy in which type $t_{1}$ chooses message $k$ and type $t_{2}$ still sends message $m$. 


\section{Relation with sequential equilibrium}

In this section we investigate the relation between preference conjecture equilibria and sequential equilibria in a signaling game.

Consider a conjecture profile

$$
c=\left(c_{12},\left(c_{21}(m)\right)_{m \in M^{*}}\right)=\left(\mu_{12}, u_{12},\left(\mu_{21}(m), u_{21}(m)\right)_{m \in M^{*}}\right) .
$$

Is there an intuitive way to associate with $c$ an assessment $\left(\sigma_{1}, \sigma_{2}, \beta\right)$ ? Note that, for example, $\mu_{21}\left(h_{0}\right)$ is player 2's conjecture at the beginning of the game about player 1's strategy choice. Since we assumed common belief of conjectures among players, we know that player 1's (informal) conjecture about $\mu_{21}\left(h_{0}\right)$ coincides with $\mu_{21}\left(h_{0}\right)$. Hence, for equilibrium analysis it seems intuitive to put $\sigma_{1}$ equal to $\mu_{21}\left(h_{0}\right)$. In other words, if we interpret $\sigma_{1}$ as the strategy that player 1 actually plays, then this is equal to what player 1 thinks that player 2 thinks that player 1 plays. By a similar line of reasoning it seems intuitive to put $\sigma_{2}$ equal to $\mu_{12}$. Thus,

$$
\sigma_{1}:=\mu_{21}\left(h_{0}\right), \quad \sigma_{2}:=\mu_{12} .
$$

Also, for the beliefs $\beta$ held by player 2 , it is natural to let

$$
\beta(t \mid m):=\frac{\sum_{s_{1}: s_{1}(t)=m} p(t) \cdot \mu_{21}(m)\left(s_{1}\right)}{\sum_{t^{\prime} \in T} \sum_{s_{1}: s_{1}\left(t^{\prime}\right)=m} p\left(t^{\prime}\right) \cdot \mu_{21}(m)\left(s_{1}\right)} \text { for all } t \in T \text { and } m \in M
$$

(note that the denominator is always positive). We now say that the assessment $\left(\sigma_{1}, \sigma_{2}, \beta\right)$, defined by (2) and (3), is induced by the conjecture profile $c$. One may note that, informally speaking, the assumption of common belief in the conjecture profile $c$ justifies the usual assumption of common knowledge for the induced assessment $\left(\sigma_{1}, \sigma_{2}, \beta\right)$.

Theorem 4.1 Let $\mathcal{S}=\left(T, M, A, p, u_{1}, u_{2}\right)$ be a signaling game and let $c$ be a preference conjecture equilibrium in $\mathcal{S}$ with $u_{21}\left(h_{0}\right)=u_{1}$ and $u_{12}=u_{2}$. Then the assessment induced by $c$ is a sequential equilibrium in $\mathcal{S}$.

Proof. We first show that $\sigma_{1}$ is optimal with respect to $\sigma_{2}$. Let $s_{1} \in S_{1}$ with $\sigma_{1}\left(s_{1}\right)>0$, hence $\mu_{21}\left(h_{0}\right)\left(s_{1}\right)>0$ by $(2)$. Optimality of $c$ implies

$$
U_{21}\left(t, s_{1}, u_{21}\left(h_{0}\right), \mu_{12}\right) \geq U_{21}\left(t, s_{1}^{\prime}, u_{21}\left(h_{0}\right), \mu_{12}\right)
$$


for all $s_{1}^{\prime} \in S_{1}$ and $t \in T$. With $u_{21}\left(h_{0}\right)=u_{1}$ and $\sigma_{2}=\mu_{12}$ this implies

$$
\sum_{s_{2} \in S_{2}} \sigma_{2}\left(s_{2}\right) \cdot u_{1}\left(t, s_{1}(t), s_{2}\left(s_{1}(t)\right)\right) \geq \sum_{s_{2} \in S_{2}} \sigma_{2}\left(s_{2}\right) \cdot u_{1}\left(t, s_{1}^{\prime}(t), s_{2}\left(s_{1}^{\prime}(t)\right)\right)
$$

for all $s_{1}^{\prime} \in S_{1}$ and $t \in T$. Hence

$$
U_{1}\left(t, s_{1}, \sigma_{2}\right) \geq U_{1}\left(t, s_{1}^{\prime}, \sigma_{2}\right)
$$

for all $s_{1}^{\prime} \in S_{1}$ and $t \in T$. So $\sigma_{1}$ is optimal with respect to $\sigma_{2}$.

Next, we show that $\sigma_{2}$ is optimal with respect to $\beta$. Let $s_{2} \in S_{2}$ with $\sigma_{2}\left(s_{2}\right)>0$, hence $\mu_{12}\left(s_{2}\right)>0$ by $(2)$. Optimality of $c$ implies

$$
U_{12}\left(m, s_{2}, u_{12}, \mu_{21}(m)\right) \geq U_{12}\left(m, s_{2}^{\prime}, u_{12}, \mu_{21}(m)\right)
$$

for all $s_{2}^{\prime} \in S_{2}$ and $m \in M$. Since, by (3), for all $m \in M$ and $s_{2}^{\prime} \in S_{2}$,

$$
\begin{aligned}
U_{12}\left(m, s_{2}^{\prime}, u_{12}, \mu_{21}(m)\right) & \\
= & \sum_{t \in T}\left[\sum_{s_{1}: s_{1}(t)=m} p(t) \cdot \mu_{21}(m)\left(s_{1}\right)\right] \cdot u_{12}\left(t, m, s_{2}^{\prime}(m)\right) \\
& =\sum_{t \in T} \beta(t \mid m)\left[\sum_{t^{\prime} \in T} \sum_{s_{1}: s_{1}\left(t^{\prime}\right)=m} p\left(t^{\prime}\right) \cdot \mu_{21}(m)\left(s_{1}\right)\right] \cdot u_{2}\left(t, m, s_{2}^{\prime}(m)\right) \\
& =\left[\sum_{t^{\prime} \in T} \sum_{s_{1}: s_{1}\left(t^{\prime}\right)=m} p\left(t^{\prime}\right) \cdot \mu_{21}(m)\left(s_{1}\right)\right] \cdot U_{2}\left(m, s_{2}^{\prime}, \beta\right)
\end{aligned}
$$

and the last term in brackets is always positive, (4) implies

$$
U_{2}\left(m, s_{2}, \beta\right) \geq U_{2}\left(m, s_{2}^{\prime}, \beta\right)
$$

for all $m \in M$ and all $s_{2}^{\prime} \in S_{2}$. Therefore, $\sigma_{2}$ is optimal with respect to $\beta$.

Finally, we show Bayesian consistency of $\left(\sigma_{1}, \beta\right)$. Let $m \in M$ such that $\sum_{t^{\prime} \in T} p\left(t^{\prime}\right) \sigma_{1}\left(m \mid t^{\prime}\right)>0$. Then there exists a $t^{\prime}$ with $\sigma_{1}\left(m \mid t^{\prime}\right)>0$, hence 
$\sum_{s_{1}^{\prime} \in S_{1}(m)} \mu_{21}\left(h_{0}\right)\left(s_{1}^{\prime}\right)=\sum_{s_{1}^{\prime} \in S_{1}(m)} \sigma_{1}\left(s_{1}^{\prime}\right)>0$. Thus, for every $t \in T$,

$$
\begin{aligned}
\beta(t \mid m) & =\frac{\sum_{s_{1}: s_{1}(t)=m} p(t) \cdot \mu_{21}(m)\left(s_{1}\right)}{\sum_{t^{\prime} \in T} \sum_{s_{1}: s_{1}\left(t^{\prime}\right)=m} p\left(t^{\prime}\right) \cdot \mu_{21}(m)\left(s_{1}\right)} \\
& =\frac{\sum_{s_{1}: s_{1}(t)=m} p(t) \cdot \mu_{21}\left(h_{0}\right)\left(s_{1}\right)}{\sum_{t^{\prime} \in T} \sum_{s_{1}: s_{1}(t)=m} p\left(t^{\prime}\right) \cdot \mu_{21}\left(h_{0}\right)\left(s_{1}\right)} \\
& =\frac{\sum_{s_{1}: s_{1}(t)=m} p(t) \cdot \sigma_{1}\left(s_{1}\right)}{\sum_{t^{\prime} \in T} \sum_{s_{1}: s_{1}(t)=m} p\left(t^{\prime}\right) \cdot \sigma_{1}\left(s_{1}\right)} \\
& =\frac{p(t) \cdot \sigma_{1}(m \mid t)}{\sum_{t^{\prime} \in T} p\left(t^{\prime}\right) \cdot \sigma_{1}\left(m \mid t^{\prime}\right)},
\end{aligned}
$$

where the first equality follows from (3) and the second equality from Bayesian updating of $c$. Hence, $\left(\sigma_{1}, \beta\right)$ is Bayesian consistent.

Example 4.2 To illustrate this, consider again the game in Figure 2 and the preference conjecture equilibrium $c$ discussed in Example 3.6. The sequential equilibrium $\left(\sigma_{1}, \sigma_{2}, \beta\right)$ induced by this preference conjecture equilibrium has $\sigma_{1}=(l, m), \sigma_{2}=(a, a, a), \beta(k)=(1,0), \beta(l)=(1,0)$ and $\beta(m)=(0,1)$.

The next theorem shows that, in turn, every sequential equilibrium corresponds to a preference conjecture equilibrium.

Theorem 4.3 Let $\mathcal{S}=\left(T, M, A, p, u_{1}, u_{2}\right)$ be a signaling game and let the assessment $\left(\sigma_{1}, \sigma_{2}, \beta\right)$ be a sequential equilibrium. Then there exists a preference conjecture equilibrium $c$ with $u_{21}\left(h_{0}\right)=u_{1}$ and $u_{12}=u_{2}$ that induces $\left(\sigma_{1}, \sigma_{2}, \beta\right)$.

Proof. We start with constructing a conjecture profile $c$. Define $\mu_{21}\left(h_{0}\right):=$ $\sigma_{1}$ and $\mu_{12}:=\sigma_{2}$. Let $m \in M$. For the definition of $\mu_{21}(m)$ we distinguish two cases:

Case 1: there exists an $s_{1}^{\prime} \in S_{1}(m)$ such that $\sigma_{1}\left(s_{1}^{\prime}\right)>0$. Then we define

$$
\mu_{21}(m)\left(s_{1}\right):=\frac{\mu_{21}\left(h_{0}\right)\left(s_{1}\right)}{\sum_{s_{1}^{\prime} \in S_{1}(m)} \mu_{21}\left(h_{0}\right)\left(s_{1}^{\prime}\right)} \text { for all } s_{1} \in S_{1}(m) .
$$

Case 2: $\sigma_{1}\left(s_{1}^{\prime}\right)=0$ for all $s_{1}^{\prime} \in S_{1}(m)$. Choose for every $t \in T$ a strategy $s_{1}^{t, m} \in S_{1}(m)$ such that $s_{1}^{t, m}(t)=m$ and $s_{1}^{t, m}\left(t^{\prime}\right) \neq m$ for all $t^{\prime} \neq t$ and define

$$
\widetilde{\mu}_{21}(m)\left(s_{1}^{t, m}\right):=\frac{\beta(t \mid m)}{p(t)}
$$


for all $t \in T$ and

$$
\tilde{\mu}_{21}(m)\left(s_{1}\right)=0
$$

for all $s_{1} \in S_{1}(m)$ with $s_{1} \neq s_{1}^{t, m}$ for all $t \in T$. Next normalize for all $s_{1} \in S_{1}(m)$ :

$$
\mu_{21}(m)\left(s_{1}\right)=\frac{\widetilde{\mu}_{21}(m)\left(s_{1}\right)}{\sum_{s_{1}^{\prime} \in S_{1}(m)} \widetilde{\mu}_{21}(m)\left(s_{1}^{\prime}\right)},
$$

which is well-defined since there exist $s_{1}^{\prime} \in S_{1}(m)$ such that $\tilde{\mu}_{21}(m)\left(s_{1}^{\prime}\right) \neq 0$, namely those strategies $s_{1}^{t, m}$ with $\beta(t \mid m)>0$.

Before completing the construction of $c$ we show that the ingredients defined thus far induce $\left(\sigma_{1}, \sigma_{2}, \beta\right)$. For this we still have to prove (3). Let $t \in T$ and $m \in M$. If case 1 above applies, then

$$
\begin{aligned}
\beta(t \mid m) & =\frac{p(t) \cdot \sigma_{1}(m \mid t)}{\sum_{t^{\prime} \in T} p\left(t^{\prime}\right) \cdot \sigma_{1}\left(m \mid t^{\prime}\right)} \\
& =\frac{p(t) \cdot \sum_{s_{1}: s_{1}(t)=m} \sigma_{1}\left(s_{1}\right)}{\sum_{t^{\prime} \in T} p\left(t^{\prime}\right) \cdot \sum_{s_{1}: s_{1}\left(t^{\prime}\right)=m} \sigma_{1}\left(s_{1}\right)} \\
& =\frac{p(t) \cdot \sum_{s_{1}: s_{1}(t)=m} \mu_{21}\left(h_{0}\right)\left(s_{1}\right)}{\sum_{t^{\prime} \in T} p\left(t^{\prime}\right) \cdot \sum_{s_{1}: s_{1}\left(t^{\prime}\right)=m} \mu_{21}\left(h_{0}\right)\left(s_{1}\right)} \\
& =\frac{\sum_{s_{1}: s_{1}(t)=m} p(t) \cdot \mu_{21}(m)\left(s_{1}\right)}{\sum_{t^{\prime} \in T} \sum_{s_{1}: s_{1}\left(t^{\prime}\right)=m} p\left(t^{\prime}\right) \mu_{21}(m)\left(s_{1}\right)},
\end{aligned}
$$

so (3) holds. If case 2 above applies, then

$$
\begin{aligned}
\frac{\sum_{s_{1}: s_{1}(t)=m} p(t) \cdot \mu_{21}(m)\left(s_{1}\right)}{\sum_{t^{\prime} \in T} \sum_{s_{1}: s_{1}\left(t^{\prime}\right)=m} p\left(t^{\prime}\right) \cdot \mu_{21}(m)\left(s_{1}\right)} & =\frac{\sum_{s_{1}: s_{1}(t)=m} p(t) \cdot \tilde{\mu}_{21}(m)\left(s_{1}\right)}{\sum_{t^{\prime} \in T} \sum_{s_{1}: s_{1}\left(t^{\prime}\right)=m} p\left(t^{\prime}\right) \cdot \tilde{\mu}_{21}(m)\left(s_{1}\right)} \\
& =\frac{\beta(t \mid m)}{\sum_{t^{\prime} \in T} \beta\left(t^{\prime} \mid m\right)} \\
& =\beta(t \mid m),
\end{aligned}
$$

so also in this case (3) holds. Hence, $c$ induces $\left(\sigma_{1}, \sigma_{2}, \beta\right)$.

We now complete the construction of $c$. Let $u_{12}:=u_{2}, u_{21}\left(h_{0}\right):=u_{1}$, and for all $t \in T, m, m^{\prime} \in M$, and $a \in A$ let

$$
u_{21}(m)\left(t, m^{\prime}, a\right):=0 .
$$


In order to show that $c$ is a preference conjecture equilibrium first note that it satisfies Bayesian updating: for $m \in M$ such that $\sum_{s_{1}^{\prime} \in S_{1}(m)} \mu_{21}\left(h_{0}\right)\left(s_{1}^{\prime}\right)>$ 0 , case 1 above applies, so Bayesian updating follows immediately.

Finally, we show optimality of $c$. Let $s_{1} \in S_{1}$. If $\mu_{21}\left(h_{0}\right)\left(s_{1}\right)>0$ then $\sigma_{1}\left(s_{1}\right)>0$, so optimality of $\sigma_{1}$ with respect to $\sigma_{2}$ yields that $s_{1}$ is optimal with respect to $\sigma_{2}$ in $\mathcal{S}$. Hence $s_{1}$ is optimal with respect to $\mu_{12}$ and $u_{21}\left(h_{0}\right)$. Further, for every $m \in M, s_{1}$ is optimal with respect to $\mu_{12}$ and $u_{21}(m)$ since player 1 is indifferent between all messages $m$ by definition of $u_{21}(m)$. This shows part 1 of Definition 3.3, i.e., optimality on the part of player 1 .

Let $s_{2} \in S_{2}$ such that $\mu_{12}\left(s_{2}\right)>0$. Then $\sigma_{2}\left(s_{2}\right)>0$ and optimality of $\sigma_{2}$ with respect to $\beta$ implies that $s_{2}$ is optimal with respect to $\beta$ on any $m \in M$, that is

$$
U_{2}\left(m, s_{2}, \beta\right) \geq U_{2}\left(m, s_{2}^{\prime}, \beta\right)
$$

for all $s_{2}^{\prime} \in S_{2}$ and all $m \in M$. Since

$$
U_{2}\left(m, s_{2}, \beta\right)=\sum_{t \in T} \beta(t \mid m) \cdot u_{2}\left(t, m, s_{2}(m)\right)
$$

(5) implies, together with (3) and $u_{12}=u_{2}$

$$
\begin{aligned}
U_{12}\left(m, s_{2}, u_{12}, \mu_{21}(m)\right) & =\sum_{t \in T} p(t) \sum_{s_{1}: s_{1}(t)=m} \mu_{21}(m)\left(s_{1}\right) \cdot u_{12}\left(t, m, s_{2}(m)\right) \\
& \geq \sum_{t \in T} p(t) \sum_{s_{1}: s_{1}(t)=m} \mu_{21}(m)\left(s_{1}\right) \cdot u_{12}\left(t, m, s_{2}^{\prime}(m)\right) \\
& =U_{12}\left(m, s_{2}^{\prime}, u_{12}, \mu_{21}(m)\right)
\end{aligned}
$$

for all $s_{2}^{\prime} \in S_{2}$ and $m \in M$. Hence, $s_{2}$ is optimal for player 2 with respect to $\mu_{21}(m)$ and $u_{12}$. This completes the proof.

Theorem 4.3 implies in particular that a preference conjecture equilibrium always exists.

\section{Minimum revision}

In a preference conjecture equilibrium player 2 can revise his conjecture about the utilities and strategies of player 1. Intuitively, one would like to keep these revisions as limited as possible. In this section we propose a way to measure this, and we study the resulting refinement of preference conjecture equilibrium and the associated refinement of sequential equilibrium. 
The measure which we are going to use is based on the number of "utility changes' player 2 has to make in order to rationalize player 1's move. Before elaborating on this we need some notations and definitions.

A (weak) ordering on a finite set $E$ is a complete and transitive binary relation on $E$. Generically, for an ordering $R$ we denote by $P$ and $I$ its asymmetric and symmetric parts, respectively. For $x, y \in E$, the expressions $x R y, x P y$, and $x I y$, are interpreted as ' $x$ is weakly preferred to $y$ ', ' $x$ is strictly preferred to $y$ ', and ' $x$ is indifferent to $y$ '. Let $R$ and $R^{\prime}$ be two orderings. We define

$$
d(\{x, y\}):= \begin{cases}1 & \text { if }\left[x P y \text { and not } x P^{\prime} y\right] \\ & \text { or }\left[x I y \text { and not } x I^{\prime} y\right] \\ & \text { or }\left[y P x \text { and not } y P^{\prime} x\right] \\ 0 & \text { otherwise, }\end{cases}
$$

and we define the distance between $R$ and $R^{\prime}$ as

$$
d\left(R, R^{\prime}\right):=\frac{1}{2} \sum_{x, y \in E} d(\{x, y\}) .
$$

Thus, the distance between two orderings is defined as the number of pairs of elements that are ranked differently in the two orderings.

\subsection{Minimum revision equilibrium}

Consider a conjecture profile $c=\left(\mu_{12}, u_{12},\left(\mu_{21}(m), u_{21}(m)\right)_{m \in M^{*}}\right)$. Player 2 may revise his conjecture about player 1's utility function and thus, for each type $t$, his conjecture about $t$ 's ordering of all messages $m \in M$ in terms of expected utility. For $t \in T, m \in M$, and $m^{\prime} \in M^{*}$ it will be convenient to use the notation

$$
U_{21}\left(t, m, u_{21}\left(m^{\prime}\right), \mu_{12}\right):=\sum_{s_{2} \in S_{2}} \mu_{12}\left(s_{2}\right) \cdot u_{21}\left(m^{\prime}\right)\left(t, m, s_{2}(m)\right)
$$

for player 2's conjecture of the expected utility for type $t$ of player 1 of playing $m$ at the beginning of the game $\left(m^{\prime}=h_{0}\right)$ or after observing $m^{\prime} \in M$. By $R_{m^{\prime}}^{t}\left(m^{\prime} \in M^{*}\right)$ we denote the (preference) ordering on $M$ for type $t$ induced by these conjectured expected utilities, i.e.,

for all $m, l \in M: m R_{m^{\prime}}^{t} l \Leftrightarrow U_{21}\left(t, m, u_{21}\left(m^{\prime}\right), \mu_{12}\right) \geq U_{21}\left(t, l, u_{21}\left(m^{\prime}\right), \mu_{12}\right)$.

The distance between two orderings $R_{m}^{t}$ and $R_{h_{0}}^{t}$ is indicative of the number of 'utility changes' player 2 makes for type $t$ of player 1 if he observes message $m$. This leads to the following definition. 
Definition 5.1 The revision index of the conjecture profile $c$ is the number

$$
d(c):=\sum_{m \in M} \sum_{t \in T} d\left(R_{h_{0}}^{t}, R_{m}^{t}\right) .
$$

Example 5.2 Consider again the signaling game of Example 2.2, see Figure 2. Let $c$ be the conjecture profile such that $\mu_{12}=(a, b, b)$ and $u_{12}$ is equal to the depicted utility function. Furthermore, $\mu_{21}\left(h_{0}\right)=\mu_{21}(k)=\mu_{21}(m)=$ $(k, m)$ and $\mu_{21}(l)=(k, l)$. Finally, $u_{21}\left(h_{0}\right), u_{21}(k)$ and $u_{21}(m)$ are equal to the depicted utility function, $u_{21}(l)\left(t_{2}, l, b\right)=4, u_{21}(l)\left(t_{2}, k, a\right)=3$ and $u_{21}(l)\left(t, m^{\prime}, a^{\prime}\right)=u_{21}\left(h_{0}\right)\left(t, m^{\prime}, a^{\prime}\right)$ otherwise. Then, $R_{h_{0}}^{t_{2}}$ orders $m$ strictly above $l$ and $l$ strictly above $k$; and $R_{l}^{t_{2}}$ orders $l$ strictly above $k$ and $k$ strictly above $m$. Hence, $d\left(R_{h_{0}}^{t_{2}}, R_{l}^{t_{2}}\right)=2$.

By requiring that the revision index be as small as possible, we obtain the announced refinement of preference conjecture equilibrium.

Definition 5.3 Let $c$ be a preference conjecture equilibrium with $u_{12}=u_{2}$ and $u_{21}\left(h_{0}\right)=u_{1}$. Then $c$ is a minimum revision equilibrium if

$$
d(c) \leq d\left(c^{\prime}\right)
$$

for all preference conjecture equilibria $c^{\prime}$ with $u_{12}^{\prime}=u_{2}$ and $u_{21}^{\prime}\left(h_{0}\right)=u_{1}$.

Since - as a consequence of Theorem 4.3-a preference conjecture equilibrium always exists, it follows that a minimum revision equilibrium always exists.

\subsection{Relation with sequential equilibrium}

A minimum revision equilibrium induces a sequential equilibrium (Theorem 4.1). Since for every sequential equilibrium there is a prefence conjecture equilibrium with $u_{1}=u_{21}\left(h_{0}\right)$ and $u_{2}=u_{12}$ that induces it (Theorem 4.3), the concept of minimum revision equilibrium can be used to obtain a refinement of sequential equilibrium. In this subsection we explore the relationship between minimum revision equilibrium and sequential equilibrium.

We will use the notation

$$
U_{1}\left(t, m, \sigma_{2}\right):=\sum_{s_{2} \in S_{2}} \sigma_{2}\left(s_{2}\right) \cdot u_{1}\left(t, m, s_{2}(m)\right)
$$

for the expected utility for player 1 if his type is $t \in T$, he chooses message $m \in M$, and player 2 plays $\sigma_{2} \in \Delta\left(S_{2}\right)$. For a finite set $E,|E|$ denotes the number of elements of $E$. 
Definition 5.4 Let $\left(\sigma_{1}, \sigma_{2}, \beta\right)$ be a sequential equilibrium of $\mathcal{S}$. Denote

$$
\begin{aligned}
R\left(\sigma_{1}, \sigma_{2}, \beta\right):=\left\{\left(t, m, m^{\prime}\right) \in\right. & T \times M \times M \mid \beta(t \mid m)>0 \\
& \text { and } \left.U_{1}\left(t, m, \sigma_{2}\right)<U_{1}\left(t, m^{\prime}, \sigma_{2}\right)\right\} .
\end{aligned}
$$

Then

$$
r\left(\sigma_{1}, \sigma_{2}, \beta\right):=|R(\sigma, \beta)|
$$

is the revision index of $\left(\sigma_{1}, \sigma_{2}, \beta\right)$.

If a triple $\left(t, m, m^{\prime}\right)$ is in $R\left(\sigma_{1}, \sigma_{2}, \beta\right)$, then player 2 believes that type $t$ has positive probability although the observed message $m$ is not optimal for this type. If player 2 believes that player 1 is rational, then he should make some 'revision' in order to rationalize this. This explains the term 'revision index' in Definition 5.4. The following theorem justifies the use of this particular expression.

Theorem 5.5 Let $\left(\sigma_{1}, \sigma_{2}, \beta\right)$ be a sequential equilibrium in the signaling game $\mathcal{S}=\left(T, M, A, p, u_{1}, u_{2}\right)$. Then:

(a) if c is a preference conjecture equilibrium with $u_{12}=u_{2}$ and $u_{21}\left(h_{0}\right)=$ $u_{1}$ which induces $\left(\sigma_{1}, \sigma_{2}, \beta\right)$, then

$$
d(c) \geq r\left(\sigma_{1}, \sigma_{2}, \beta\right) .
$$

(b) there exists a preference conjecture equilibrium c with $u_{12}=u_{2}$ and $u_{21}\left(h_{0}\right)=u_{1}$ which induces $\left(\sigma_{1}, \sigma_{2}, \beta\right)$, such that

$$
d(c)=r\left(\sigma_{1}, \sigma_{2}, \beta\right) .
$$

Proof. (a) Let $c$ be a preference conjecture equilibrium with $u_{12}=u_{2}$ and $u_{21}\left(h_{0}\right)=u_{1}$ which induces $\left(\sigma_{1}, \sigma_{2}, \beta\right)$. Let $t \in T$ and $m \in M$. If, for an $m^{\prime} \in M,\left(t, m, m^{\prime}\right) \in R(\sigma, \beta)$, then $\beta(t \mid m)>0$. Hence, by (3), there exists an $s_{1} \in S_{1}$ such that $s_{1}(t)=m$ and $\mu_{21}(m)\left(s_{1}\right)>0$. Optimality of $c$ implies

$$
U_{21}\left(t, m, u_{21}(m), \mu_{12}\right) \geq U_{21}\left(t, m^{\prime}, u_{21}(m), \mu_{12}\right) .
$$

Thus, $R_{m}^{t}$ orders $m$ weakly above $m^{\prime}$. On the other hand,

$$
U_{1}\left(t, m, \sigma_{2}\right)<U_{1}\left(t, m^{\prime}, \sigma_{2}\right)
$$

since $\left(t, m, m^{\prime}\right) \in R(\sigma, \beta)$. This is equivalent to

$$
U_{21}\left(t, m, u_{21}\left(h_{0}\right), \mu_{12}\right)<U_{21}\left(t, m^{\prime}, u_{21}\left(h_{0}\right), \mu_{12}\right) .
$$


Hence $R_{h_{0}}^{t}$ orders $m$ strictly below $m^{\prime}$. Thus we have

$$
d\left(R_{h_{0}}^{t}, R_{m}^{t}\right) \geq \mid\left\{m^{\prime} \in M \mid \text { and } U_{1}\left(t, m, \sigma_{2}\right)<U_{1}\left(t, m^{\prime}, \sigma_{2}\right)\right\} \mid,
$$

which, after summation over $t \in T$ and $m \in M$, yields

$$
\begin{aligned}
d(c) & =\sum_{m \in M} \sum_{t \in T} d\left(R_{h_{0}}^{t}, R_{m}^{t}\right) \\
& \geq \sum_{(t, m): \beta(t \mid m)>0}\left|\left\{m^{\prime} \in M \mid U_{1}\left(t, m, \sigma_{2}\right)<U_{1}\left(t, m^{\prime}, \sigma_{2}\right)\right\}\right| \\
& =r\left(\sigma_{1}, \sigma_{2}, \beta\right) .
\end{aligned}
$$

(b) In order to construct a $c$ with the desired properties, start with $u_{21}\left(h_{0}\right):=u_{1}, u_{12}:=u_{2}, \mu_{21}\left(h_{0}\right):=\sigma_{1}$ and $\mu_{12}:=\sigma_{2}$. Let $t \in T$ and $m \in M$. If there exists an $m^{\prime} \in M$ with $\left(t, m, m^{\prime}\right) \in R\left(\sigma_{1}, \sigma_{2}, \beta\right)$, we define

$$
u_{21}(m)(t, m, a):=\max _{m^{\prime \prime}} u_{21}\left(h_{0}\right)\left(t, m^{\prime \prime}, a\right)+1
$$

and

$$
u_{21}(m)(t, \tilde{m}, a):=u_{21}\left(h_{0}\right)(t, \tilde{m}, a)
$$

for all $\tilde{m} \neq m$ and $a \in A$. If there exists no $m^{\prime}$ with $\left(t, m, m^{\prime}\right) \in R\left(\sigma_{1}, \sigma_{2}, \beta\right)$, we define

$$
u_{21}(m)(t, \tilde{m}, a)=u_{21}\left(h_{0}\right)(t, \tilde{m}, a)
$$

for all $\tilde{m} \in M$ and all $a \in A$.

The ingredients of $c$ defined thus far are sufficient to compute $d(c)$. Let $t \in T$ and $m \in M$. If there is no $m^{\prime}$ with $\left(t, m, m^{\prime}\right) \in R\left(\sigma_{1}, \sigma_{2}, \beta\right)$, then $u_{21}(m)=u_{21}\left(h_{0}\right)$ and therefore

$$
d\left(R_{h_{0}}^{t}, R_{m}^{t}\right)=0 .
$$

Now suppose there are $m^{\prime} \in M$ with $\left(t, m, m^{\prime}\right) \in R\left(\sigma_{1}, \sigma_{2}, \beta\right)$. This means that $\beta(t \mid m)>0$, and that $U_{1}\left(t, m, \sigma_{2}\right)<U_{1}\left(t, m^{\prime}, \sigma_{2}\right)$, hence

$$
\sum_{s_{2} \in S_{2}} \mu_{12}\left(s_{2}\right) u_{21}\left(h_{0}\right)\left(t, m, s_{2}(m)\right)<\sum_{s_{2} \in S_{2}} \mu_{12}\left(s_{2}\right) u_{21}\left(h_{0}\right)\left(t, m^{\prime}, s_{2}\left(m^{\prime}\right)\right)
$$

for all such $m^{\prime} \in M$. So $R_{h_{0}}^{t}$ orders $m$ strictly below all such $m^{\prime}$, whereas, by definition of $u_{21}(m), R_{m}^{t}$ orders $m$ above all these $m^{\prime}$. Since the ordering between any two messages unequal to $m$ does not change between $R_{h_{0}}^{t}$ and $R_{m}^{t}$ (due to the definition of $u_{21}(m)$ ), we obtain:

$$
d\left(R_{h_{0}}^{t}, R_{m}^{t}\right)=\left|\left\{m^{\prime} \in M \mid U_{1}\left(t, m, \sigma_{2}\right)<U_{1}\left(t, m^{\prime}, \sigma_{2}\right)\right\}\right| .
$$


Summation over all messages $m$ and all types $t$ yields

$$
\begin{aligned}
r\left(\sigma_{1}, \sigma_{2}, \beta\right) & =\sum_{(t, m): \beta(t \mid m)>0}\left|\left\{m^{\prime} \in M \mid U_{1}\left(t, m, \sigma_{2}\right)<U_{1}\left(t, m^{\prime}, \sigma_{2}\right)\right\}\right| \\
& =\sum_{(t, m): \beta(t \mid m)>0} d\left(R_{h_{0}}^{t}, R_{m}^{t}\right) \\
& =\sum_{(t, m): \beta(t \mid m)>0} d\left(R_{h_{0}}^{t}, R_{m}^{t}\right)+\sum_{(t, m): \beta(t \mid m)=00} d\left(R_{h_{0}}^{t}, R_{m}^{t}\right) \\
& =d(c) .
\end{aligned}
$$

We are left to show that $c$ can be extended to a preference conjecture equilibrium. Let $m \in M$ : we still have to define $\mu_{21}(m)$. We distinguish the same two cases as in the proof of Theorem 4.3.

Case 1: there exists an $s_{1}^{\prime} \in S_{1}(m)$ such that $\sigma_{1}\left(s_{1}^{\prime}\right)>0$. Then we define

$$
\mu_{21}(m)\left(s_{1}\right)=\frac{\mu_{21}\left(h_{0}\right)\left(s_{1}\right)}{\sum_{s_{1}^{\prime} \in S_{1}(m)} \mu_{21}\left(h_{0}\right)\left(s_{1}^{\prime}\right)} \text { for all } s_{1} \in S_{1}(m) .
$$

Case 2: $\sigma_{1}\left(s_{1}^{\prime}\right)=0$ for all $s_{1}^{\prime} \in S_{1}(m)$. Choose for every $t \in T$ a strategy $s_{1}^{t, m} \in S_{1}$ such that (i) $s_{1}^{t, m}(t)=m$ and (ii) for all $t^{\prime} \neq t, s_{1}^{t, m}\left(t^{\prime}\right) \neq m$ and $s_{1}^{t, m}\left(t^{\prime}\right)$ is optimal for $t^{\prime}$ with respect to $\mu_{12}$ and $u_{21}\left(h_{0}\right)$. The latter is always possible since $\sigma_{1}$ is optimal with respect to $\sigma_{2}$ and $\sigma_{1}\left(s_{1}\right)=0$ for all $s_{1} \in S_{1}(m)$. Now define

$$
\widetilde{\mu}_{21}(m)\left(s_{1}^{t, m}\right):=\frac{\beta(t \mid m)}{p(t)}
$$

for all $t \in T$ and

$$
\tilde{\mu}_{21}(m)\left(s_{1}\right)=0
$$

for all $s_{1} \in S_{1}(m)$ with $s_{1} \neq s_{1}^{t, m}$ and for all $t \in T$. Next normalize for all $s_{1} \in S_{1}(m)$ :

$$
\mu_{21}(m)\left(s_{1}\right)=\frac{\widetilde{\mu}_{21}(m)\left(s_{1}\right)}{\sum_{s_{1}^{\prime} \in S_{1}(m)} \widetilde{\mu}_{21}(m)\left(s_{1}^{\prime}\right)} .
$$

This completes the definition of $c$. In the proof of Theorem 4.3 we already showed that this $c$ induces $\left(\sigma_{1}, \sigma_{2}, \beta\right)$; that it satisfies Bayesian updating; that $c$ is optimal on the part of player 2, that is, part 2 of Definition 3.3; and that $c$ is optimal on the part of player 1 on $h_{0}$. Since the definition of $u_{21}(m)(m \in M)$ is different from the one in the proof of Theorem 4.3, we 
still have to show that, for all $m \in M$ and $s_{1} \in S_{1}(m)$, if $\mu_{21}(m)\left(s_{1}\right)>0$, then $s_{1}$ is optimal for player 1 with respect to $\mu_{12}$ and $u_{21}(m)$.

Let $m \in M$. First suppose that there is an $s_{1}^{\prime} \in S_{1}(m)$ for which $\sigma_{1}\left(s_{1}^{\prime}\right)>0$, so that case 1 applies. Let $s_{1} \in S_{1}(m)$ with $\mu_{21}(m)\left(s_{1}\right)>0$. Then $\mu_{21}\left(h_{0}\right)\left(s_{1}\right)>0$. Since $\sigma_{1}$ is optimal with respect to $\sigma_{2}$ and $m$ is played with positive probability, the set

$$
\left\{\left(t, m^{\prime}\right) \in T \times M \mid \beta(t \mid m)>0 \text { and } U_{1}\left(t, m, \sigma_{2}\right)<U_{1}\left(t, m^{\prime}, \sigma_{2}\right)\right\}
$$

is empty. Thus there exists no $m^{\prime}$ such that $\left(t, m, m^{\prime}\right) \in R\left(\sigma_{1}, \sigma_{2}, \beta\right)$, which implies that $u_{21}(m)=u_{21}\left(h_{0}\right)$. In the proof of Theorem 4.3 we already showed that $\mu_{21}\left(h_{0}\right)\left(s_{1}\right)>0$ implies optimality of $s_{1}$ for player 1 with respect to $\mu_{12}$ and $u_{21}\left(h_{0}\right)$ and thus, $\mu_{21}(m)\left(s_{1}\right)>0$ implies optimality of $s_{1}$ for player 1 with respect to $\mu_{12}$ and $u_{21}(m)$.

Next suppose that $\sigma_{1}\left(s_{1}^{\prime}\right)=0$ for all $s_{1}^{\prime} \in S_{1}(m)$, so that case 2 applies. Let $s_{1} \in S_{1}(m)$ with $\mu_{21}(m)\left(s_{1}\right)>0$. This implies that $s_{1}=s_{1}^{t, m}$ for a unique $t \in T$ and that $\beta(t \mid m)>0$ for this $t$, by definition of $\tilde{\mu}_{21}(m)$ and $s_{1}^{t, m}$. We distinguish two subcases.

First, suppose that message $s_{1}(t)=m$ is not optimal for $t$ with respect to $\mu_{12}$ and $u_{21}\left(h_{0}\right)$. Then there exists an $m^{\prime} \in M$ such that $U_{1}\left(t, m, \sigma_{2}\right)<$ $U_{1}\left(t, m^{\prime}, \sigma_{2}\right)$, and therefore the set

$$
\left\{m^{\prime} \in M \mid \beta(t \mid m)>0 \text { and } U_{1}\left(t, m, \sigma_{2}\right)<U_{1}\left(t, m^{\prime}, \sigma_{2}\right)\right\}
$$

is nonempty. But then, by definition,

$$
u_{21}(m)(t, m, a)=\max _{m^{\prime \prime} \in M} u_{21}\left(h_{0}\right)\left(t, m^{\prime \prime}, a\right)+1
$$

for all $a \in A$, which implies that $m \in M$ becomes optimal for type $t$ with respect to $\mu_{12}$ and $u_{21}(m)$. By definition of $s_{1}^{t, m}$, message $s_{1}^{t, m}\left(t^{\prime}\right)$ is optimal for all $t^{\prime} \neq t$ with respect to $\mu_{12}$ and $u_{21}\left(h_{0}\right)$. Since for these $t^{\prime}$ we have $u_{21}(m)\left(t^{\prime}, m^{\prime}, a\right)=u_{21}\left(h_{0}\right)\left(t^{\prime}, m^{\prime}, a\right)$ for all $m^{\prime} \in M$ and all $a \in A$, this implies that $s_{1}^{t, m}\left(t^{\prime}\right)$ is optimal with respect to $\mu_{12}$ and $u_{21}(m)$. Thus $s_{1}=$ $s_{1}^{t, m}$ is optimal with respect to $\mu_{12}$ and $u_{21}(m)$.

Second, suppose that $s_{1}(t)=m$ is optimal for $t$ with respect to $\mu_{12}$ and $u_{21}\left(h_{0}\right)$. Then there exists no $m^{\prime}$ such that $\left(t, m, m^{\prime}\right) \in R\left(\sigma_{1}, \sigma_{2}, \beta\right)$. Hence, $u_{21}(m)\left(t, m^{\prime}, a\right)=u_{21}\left(h_{0}\right)\left(t, m^{\prime}, a\right)$ for all $m^{\prime} \in M$ and all $a \in A$, which implies that $s_{1}(t)$ is optimal with respect to $\mu_{12}$ and $u_{21}(m)$. By the same argument as in the first subcase, the strategy $s_{1}$ is optimal for all other $t^{\prime} \neq t$ with respect to $\mu_{12}$ and $u_{21}(m)$, which completes the proof. 
Definition 5.6 A sequential equilibrium $\left(\sigma_{1}, \sigma_{2}, \beta\right)$ in a signaling game is a minimum revision sequential equilibrium if it minimizes the revision index among all sequential equilibria of the game.

With this definition and Theorem 5.5 we immediately have the following corollary.

Corollary 5.7 Let $\mathcal{S}=\left(T, M, A, p, u_{1}, u_{2}\right)$ be a signaling game.

(a) If $c$ is a minimum revision equilibrium in $\mathcal{S}$, then the induced sequential equilibrium is a minimum revision sequential equilibrium.

(b) If $\left(\sigma_{1}, \sigma_{2}, \beta\right)$ is a minimum revision sequential equilibrium, then there exists a minimum revision equilibrium $c$ with $u_{21}\left(h_{0}\right)=u_{1}$ and $u_{12}=$ $u_{2}$ that induces $\left(\sigma_{1}, \sigma_{2}, \beta\right)$.

Corollary 5.7 implies the following result for the special case where every information set of player 2 is reached with positive probability.

Corollary 5.8 Let $\mathcal{S}=\left(T, M, A, p, u_{1}, u_{2}\right)$ be a signaling game. If there exists a sequential equilibrium $\left(\sigma_{1}, \sigma_{2}, \beta\right)$ such that for every $m \in M$ there is a type $t$ and a pure strategy $s_{1}$ with $s_{1}(t)=m$ and $\sigma_{1}\left(s_{1}\right)>0$, then every minimum revision equilibrium with $u_{21}\left(h_{0}\right)=u_{1}$ and $u_{12}=u_{2}$ has revision index 0 .

Proof. For a sequential equilibrium $\left(\sigma_{1}, \sigma_{2}, \beta\right)$ with these properties, the corresponding set $R\left(\sigma_{1}, \sigma_{2}, \beta\right)$ is empty and hence $\left(\sigma_{1}, \sigma_{2}, \beta\right)$ is a minimum revision sequential equilibrium. Thus every minimum revision equilibrium has revision index 0 .

\section{Examples and comparison to the intuitive crite- rion}

A commonly used refinement of sequential equilibrium in signaling games is the intuitive criterion (Cho and Kreps, 1987). This criterion restricts possible beliefs on information sets that are not reached with positive probability in equilibrium, as follows. Suppose a message $m$ is sent that has zero probability in equilibrium. Then we ask which type could possibly benefit from sending $m$, in the sense that there exists an action $a \in A$ such that

(i) $u_{1}(t, m, a) \geq \sum_{s_{1} \in S_{1}} \sigma_{1}\left(s_{1}\right) \sum_{s_{2} \in S_{2}} \sigma_{2}\left(s_{2}\right) u_{1}\left(t, s_{1}(t), s_{2}\left(s_{1}(t)\right)\right)$, and 
(ii) there is $\beta(\cdot \mid m) \in \Delta(T)$ such that $a \in \arg \max _{a^{\prime} \in A} \sum_{t^{\prime} \in T} \beta\left(t^{\prime} \mid m\right) u_{2}\left(t^{\prime}, m, a^{\prime}\right)$.

Condition (i) says that if $m$ is followed by $a$, then the utility to type $t$ is at least as high as the equilibrium utility for this type. Condition (ii) says that $a$ is a potential optimal action for player 2 after $m$.

A sequential equilibrium $\left(\sigma_{1}, \sigma_{2}, \beta\right)$ is said to survive the intuitive criterion if for every $m$ such that $(1) \sigma_{1}\left(s_{1}\right)=0$ for all $s_{1} \in S_{1}(m)$ and (2) there are types $t$ for which an $a \in A$ satisfying (i) and (ii) above exists, we have $\beta(t \mid m)>0$ only if $t$ is a type as in (2).

In this section we consider two examples of signaling games. In both examples we compute all sequential equilibria and thus, basically, all preference conjecture equilibria with the utility functions of the signaling game conjectured at the beginning (cf. Theorems 4.1 and 4.3). We also compare the refinements induced by minimum revision and intuitive criterion. In the first example there are minimum revision equilibria that survive the intuitive criterion and others that don't. The second example shows that the set of minimum revision equilibria and the set of equilibria surviving the intuitive criterion can be disjoint.

Example 6.1 See Figure 3 for the extensive form of the game and the description of all sequential equilibria. For convenience, the equilibria are described in terms of behavioral strategies. E.g., $(b, \lambda a+(1-\lambda) b)$ is the behavioral strategy where player 2 plays $b$ if player 1 plays $A$, and $a$ with probability $\lambda$ and $b$ with probability $1-\lambda$ if player 1 plays $B$. Further, $\alpha$ is the belief of player 2 that player has type $t_{1}$ if he plays $A$, and $\beta$ is the belief of player 2 that player 1 has type $t_{1}$ if he plays $B$. The revision index is denoted by $r$.

Example 6.2 This example shows that the set of minimum revision sequential equilibria and the set of sequential equilibria that survive the intuitive criterion can be disjoint. Consider the game in Figure 4, a game inspired by the beer and quiche game of Cho and Kreps (1987). Player 1 has the extra option of vegetarian quiche, which contains enough spinach for him to be stronger in case of a duel. Player 1 has two types: weak $(w)$ and strong $(s)$, with the weak type occurring with probability $\frac{1}{10}$; and three messages: beer $(b)$, quiche $(q)$ and vegetarian quiche $(v)$. Player 2 has two actions: duel $(d)$ and not duel $(n)$.

It can be shown that the only sequential equilibrium in this game that survives the intuitive criterion is the one where both types of player $1 \mathrm{drink}$ 


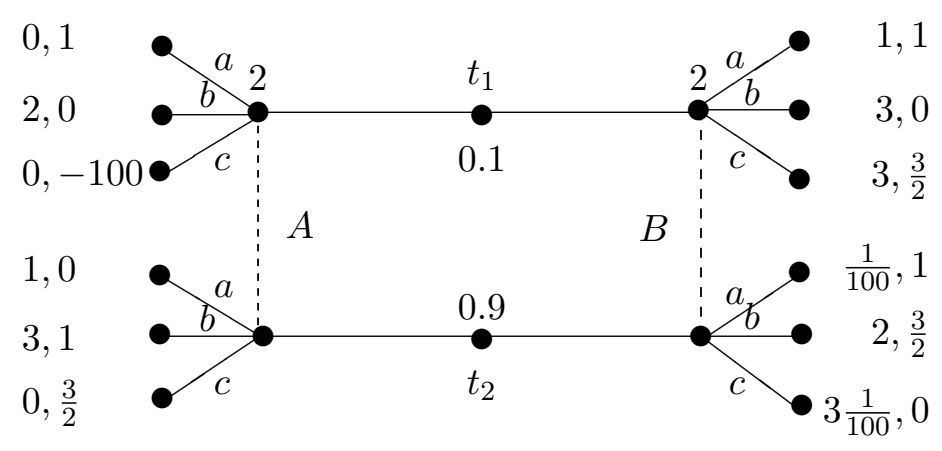

\begin{tabular}{|l|c|c|c|c|}
\hline Equilibrium (in behavioural strategies) & $\alpha$ & $\beta$ & $r$ & intuitive \\
\hline$\left\{(A, A),(b, \lambda a+(1-\lambda) b) ; \frac{1}{2}<\lambda \leq 1\right\}$ & 0.1 & $\frac{1}{3}$ & 2 & yes \\
$\left\{(A, A),\left(b, \frac{1}{2} a+\frac{1}{2} b\right)\right\}$ & 0.1 & $\frac{1}{3}$ & 1 & yes \\
$\{(A, A),(b, a)\}$ & 0.1 & $\left(\frac{1}{3}, \frac{2}{3}\right)$ & 2 & yes \\
$\left\{(A, A),(b, \lambda a+(1-\lambda) c) ; \frac{1}{2}<\lambda \leq 1\right\}$ & 0.1 & $\frac{2}{3}$ & 2 & yes \\
$\left\{(A, A),\left(b, \frac{1}{2} a+\frac{1}{2} c\right)\right\}$ & 0.1 & $\frac{2}{3}$ & 1 & yes \\
$\{(B, B),(c, b)\}$ & 0 & 0.1 & 1 & yes \\
$\{(B, B),(c, b)\}$ & $\left(0, \frac{1}{201}\right)$ & 0.1 & 2 & no \\
$\left\{(B, B),(\lambda b+(1-\lambda) c, b) ; 0<\lambda<\frac{2}{3}\right\}$ & $\frac{1}{201}$ & 0.1 & 2 & no \\
$\left\{(B, B),\left(\frac{2}{3} b+\frac{1}{3} c, b\right)\right\}$ & $\frac{1}{201}$ & 0.1 & 1 & no \\
$\{(B, B),(a, b)\}$ & 1 & 0.1 & 1 & no \\
$\{(B, B),(a, b)\}$ & $\left(\frac{1}{2}, 1\right)$ & 0.1 & 2 & no \\
$\left\{(B, B),(\lambda a+(1-\lambda) b, b) ; 0<\lambda<\frac{1}{2}\right\}$ & $\frac{1}{2}$ & 0.1 & 2 & no \\
$\left\{(B, B),\left(\frac{1}{2} a+\frac{1}{2} b, b\right)\right\}$ & $\frac{1}{2}$ & 0.1 & 1 & no \\
\hline
\end{tabular}

Figure 3: Example 6.1 


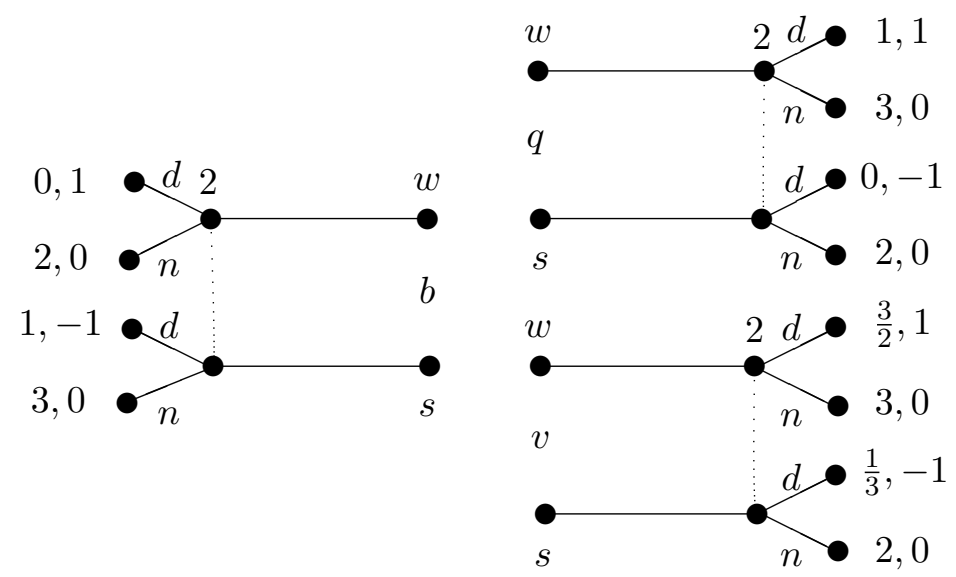

Figure 4: Example 6.2

beer, and player 2 does not duel if and only if player 1 drinks beer, and believes that player 1 is weak with probability one if he eats quiche or vegetarian quiche. So this is similar to the original beer and quiche game. This equilibrium has revision index equal to 2 and is not a minimal revision equilibrium: there are sequential equilibria that have (the minimal) revision index 1 , for instance the equilibrium where both types of player 1 eat quiche $(q)$, player 2 duels if and only if player 1 drinks beer, believes that beer signals the weak type with probability one and vegetarian quiche signals the strong type with probability greater than $1 / 2$.

\section{$7 \quad$ Structure and computation}

In this section we give a representation of the set of sequential equilibria of a signaling game as a union of subsets on which the revision index is constant. Since these subsets are polyhedral, given by systems of linear inequalities, this representation is convenient for computational purposes.

Let $I \subseteq S_{1}$ be a subset of pure strategies for player 1 and $J \subseteq S_{2}$ a subset of pure strategies for player 2. Define

$$
\begin{aligned}
U(J):= & \left\{\sigma_{1} \in \Delta\left(S_{1}\right) \mid\right. \\
& \text { all } \left.\sigma_{2} \in \Delta(J) \text { are optimal with respect to } \sigma_{1}\right\}
\end{aligned}
$$


and

$$
\begin{aligned}
U(I):= & \left\{\sigma_{2} \in \Delta\left(S_{2}\right) \mid\right. \\
& \text { all } \left.\sigma_{1} \in \Delta(I) \text { are optimal with respect to } \sigma_{2}\right\} .
\end{aligned}
$$

These sets are polytopes since all inequalities defining them are linear. As is not hard to verify (cf. Borm et al., 2002), the set of Nash equilibria of $\mathcal{S}$ is equal to

$$
N E(\mathcal{S})=\bigcup_{I \subseteq S_{1}, J \subseteq S_{2}}(\Delta(I) \cap U(J)) \times(\Delta(J) \cap U(I)) .
$$

The difference between sequential equilibrium and Nash equilibrium is that in a sequential equilibrium player 2 also plays optimally on information sets reached with zero probability (cf. Definition 2.3). Define

$$
M(I):=\left\{m \in M \mid \text { for all } t \in T \text { and } s_{1} \in I: s_{1}(t) \neq m\right\}
$$

and

$$
\begin{aligned}
B(I, J):= & \left\{\beta \in \Delta(T)^{M(I)} \mid \text { every } s_{2} \in J\right. \\
& \text { is optimal with respect to } \beta \text { on every } m \in M(I)\} .
\end{aligned}
$$

Since, in a sequential equilibrium, the beliefs of player 2 on information sets that are reached with positive probability are completely determined by Bayesian consistency, in describing such an equilibrium we only have to consider beliefs on information sets that are reached with zero probability. With some abuse of notation we therefore present a sequential equilibrium $\left(\sigma_{1}, \sigma_{2}, \beta\right)$ also as $\left(\sigma_{1}, \sigma_{2},(\beta(m))_{m \in M(I)}\right)$ with $I=\left\{s_{1} \in S_{1} \mid \sigma_{1}\left(s_{1}\right)>0\right\}$.

Lemma 7.1 The set of sequential equilibria of the signaling game $\mathcal{S}$ is equal to

$$
S E(\mathcal{S})=\bigcup_{I \subseteq S_{1}, J \subseteq S_{2}}(\Delta(I) \cap U(J)) \times(\Delta(J) \cap U(I)) \times B(I, J) .
$$

Proof. First let $\left(\sigma_{1}, \sigma_{2}, \beta\right)$ be an element of

$$
\bigcup_{I \subseteq S_{1}, J \subseteq S_{2}}(\Delta(I) \cap U(J)) \times(\Delta(J) \cap U(I)) \times B(I, J) .
$$

In view of Definition 2.3 and (6) we still have to show that every $s_{2} \in S_{2}$ with $\sigma_{2}\left(s_{2}\right)>0$ is optimal with respect to $\beta$ on every $m \in M(I)$, but this follows immediately from $s_{2} \in J$ and the definition of $B(I, J)$. 
Conversely, let $\left(\sigma_{1}, \sigma_{2}, \beta\right)$ be a sequential equilibrium (with $\beta$ only described on information sets reached with probability zero). Take $I:=\left\{s_{1} \in\right.$ $\left.S_{1} \mid \sigma_{1}\left(s_{1}\right)>0\right\}$ and $J:=\left\{s_{2} \in S_{2} \mid \sigma_{2}\left(s_{2}\right)>0\right\}$. Then, since $\left(\sigma_{1}, \sigma_{2}\right)$ is a Nash equilibrium in $\mathcal{S}$, we have

$$
\left(\sigma_{1}, \sigma_{2}\right) \in(\Delta(I) \cap U(J)) \times(\Delta(J) \cap U(I)) .
$$

Finally, since every $s_{2} \in S_{2}$ with $\sigma_{2}\left(s_{2}\right)>0$ is optimal with respect to $\beta$ on every $m$ reached with zero probability, we have $\beta \in B(I, J)$.

We will refine the representation of the set of sequential equilibria in Lemma 7.1 based on the revision index of a sequential equilibrium. Since the revision index is related to preferences of player 1 , this means that the sets $I$ in this representation have to be refined. For every type $t \in T$, let $R^{t}$ be a (for the moment arbitrary) ordering on $M$, let $R=\left(R^{t}\right)_{t \in T}$ denote a profile of such orderings, and $\mathcal{R}$ the set of all such profiles. For a mixed strategy $\sigma_{2} \in \Delta\left(S_{2}\right)$ and type $t \in T$, define the ordering $R^{t}\left(\sigma_{2}\right)$ by

$$
m R^{t}\left(\sigma_{2}\right) m^{\prime} \Leftrightarrow \sum_{s_{2} \in S_{2}} \sigma_{2}\left(s_{2}\right) u_{1}\left(t, m, s_{2}(m)\right) \geq \sum_{s_{2} \in S_{2}} \sigma_{2}\left(s_{2}\right) u_{1}\left(t, m^{\prime}, s_{2}\left(m^{\prime}\right)\right)
$$

for all $m, m^{\prime} \in M$. (Recall that this coincides with the ordering $R_{h_{0}}^{t}$ in Section 5.) The associated profile is denoted by $R\left(\sigma_{2}\right)$.

For $R \in \mathcal{R}$ define

$$
I(R):=\left\{s_{1} \in S_{1} \mid s_{1}(t) R^{t} m \text { for all } t \in T \text { and } m \in M\right\}
$$

and

$$
U(R):=\left\{\sigma_{2} \in \Delta\left(\sigma_{2}\right) \mid R\left(\sigma_{2}\right)=R\right\} .
$$

Let $a$ be a correspondence that assigns to every $m \in M(I(R))$ a nonempty subset of $T$, and let $A(R)$ denote the set of all such correspondences. For $\beta \in B(I(R), J)$ let $\bar{\beta}$ be the correspondence that assigns to every $m \in$ $M(I(R))$ the support of $\beta(m)$, i.e., $\bar{\beta}(m)=\{t \in T \mid \beta(t \mid m)>0\}$. Obviously, $\bar{\beta} \in A(R)$. Finally, let $B_{a}(I(R), J):=\{\beta \in B(I(R), J) \mid \bar{\beta}=a\}$ for every $a \in A(R)$.

The proof of the following lemma is left to the reader.

Lemma 7.2 The set of sequential equilibria in the signaling game $\mathcal{S}$ is equal to

$S E(\mathcal{S})=\bigcup_{R \in \mathcal{R}, J \subseteq S_{2}} \bigcup_{a \in A(R)}(\Delta(I(R)) \cap U(J)) \times(\Delta(J) \cap U(R)) \times B_{a}(I(R), J)$. 
The usefulness of this representation is demonstrated by the following theorem, which states that the revision index is constant on the sets in this representation.

Theorem 7.3 In the game $\mathcal{S}$, let $R \in \mathcal{R}, a \in A(R)$, and $J \subseteq S_{2}$. Let $\left(\sigma_{1}, \sigma_{2}, \beta\right)$ and $\left(\sigma_{1}^{\prime}, \sigma_{2}^{\prime}, \beta^{\prime}\right)$ be elements of

$$
(\Delta(I(R)) \cap U(J)) \times(\Delta(J) \cap U(R)) \times B_{a}(I(R), J) .
$$

Then $r\left(\sigma_{1}, \sigma_{2}, \beta\right)=r\left(\sigma_{1}^{\prime}, \sigma_{2}^{\prime}, \beta^{\prime}\right)$.

Proof. Suppose the two revision indices are different, say $r\left(\sigma_{1}, \sigma_{2}, \beta\right)<$ $r\left(\sigma_{1}^{\prime}, \sigma_{2}^{\prime}, \beta^{\prime}\right)$. Since $\bar{\beta}=\bar{\beta}^{\prime}=a$, by definition of the revision index there must be messages $m, \tilde{m} \in M(I(R))$ and a type $t \in a(m)$ such that $U_{1}\left(t, m, \sigma_{2}\right) \geq$ $U_{1}\left(t, \tilde{m}, \sigma_{2}\right)$ and $U_{1}\left(t, m, \sigma_{2}^{\prime}\right)<U_{1}\left(t, \tilde{m}, \sigma_{2}^{\prime}\right)$. This implies $R^{t}\left(\sigma_{2}\right) \neq R_{t}\left(\sigma_{2}^{\prime}\right)$ and thus $R \neq R\left(\sigma_{2}\right)$ or $R \neq R\left(\sigma_{2}^{\prime}\right)$. Thus, either $\sigma_{2} \notin U(R)$ or $\sigma_{2}^{\prime} \notin U(R)$, a contradiction.

It follows, in particular, that the set of minimum revision equilibria is the union of sets $(\Delta(I(R)) \cap U(J)) \times(\Delta(J) \cap U(I(R))) \times B_{a}(I(R), J)$ with the same (minimal) revision index.

We conclude this section with an example.

Example 7.4 Consider Example 6.1. As an illustration of Theorem 7.3, we compute one set with constant revision index. Assume that both types strictly prefer $A$ over $B$, which means that $I(R)$ contains only one element, namely $(A, A)$. It is easily seen that this implies $U(J)=\Delta(I(R))=$ $\{(A, A)\}$. Now we must choose $J$ such that all $\sigma_{2} \in \Delta(J)$ are optimal with respect to $(A, A)$. Therefore we first choose

$$
J=\{(b, a),(b, b)\},
$$

that is we choose a set of strategies that can simultaneously be optimal. The set $U(R)$ contains all mixed strategies for player 2 that make player 1 willing to play $(A, A)$. Since we have to take the intersection with $\Delta(J)$ afterwards, we only consider that part of $U(R)$ that puts positive weight only on strategies in $J$. Since we want both types to strictly prefer $A$, we obtain (the behavioral strategies) $\sigma_{2}=(b, \lambda a+(1-\lambda) b)$ with $\lambda \in\left(\frac{1}{2}, 1\right]$. Given this $I(R)$, we have $M(I(R))=\{B\}$ and we choose $a(B)=\left\{t_{1}, t_{2}\right\}$. Computation of the beliefs that make all this possible yields $\beta=\frac{1}{3}$ and we have the set of sequential equilibria

$$
\left\{(A, A),(b, \lambda a+(1-\lambda) b) ; \lambda \in\left(\frac{1}{2}, 1\right] ; \beta=\frac{1}{3}\right\}
$$

with revision index $r=2$. 


\section{References}

[1] Banks, J., and J. Sobel (1987), Equilibrium selection in signaling games, Econometrica, 55, 647-661.

[2] Borm, P., D. Vermeulen, and M. Voorneveld (2003), The structure of the set of equilibria for two person multicriteria games, European Journal of Operational Research, 148, 480-493.

[3] Cho, I.-K., and D.M. Kreps (1987), Signaling games and stable equilibria, Quarterly Journal of Economics, 102, 179-221.

[4] Grossman, S., and M. Perry (1986), Perfect sequential equilibria, Journal of Economic Theory, 39, 97-119.

[5] Harsanyi, J. (1967, 1968) Games with incomplete information played by "Bayesian" players I-III, Management Science, 14, 159-182, 320-334, 486-502.

[6] Kreps, D., and R. Wilson (1982), Sequential equilibria, Econometrica $50,863-894$.

[7] Nash, J.F. (1950), Equilibrium points in n-person games, Proceedings from the National Academy of Science, 36, 48-49.

[8] Nash, J.F. (1951), Noncooperative games, Annals of Mathematics, 54, 286-295.

[9] Perea, A. (2003), Forward induction and the minimum revision principle, revised version, University of Maastricht. 